\title{
DNA fingerprinting: A novel technique for identification of important species of citrus
}

\author{
A.H. Tupke», A.A. Akhare, S.J. Gahukar*, B.P. Bahatkar, A.R. Zadokar, P.N. Gaikwad and R.R. Katkade \\ Centre of Excellence in Plant Biotechnology, Department of Agricultural Botany, Dr. Panjabrao Deshmukh Krishi Vidhyapeeth, \\ Akola-444104, Maharashtra, India \\ *College of Food Technology, Yavatmal, Dr. Panjabrao Deshmukh Krishi Vidhyapeeth, Akola-444104, Maharashtra, India
}

\section{Article Info}

Article history

Received 8 May 2021

Revised 25 June 2021

Accepted 27 June 2021

Published online 30 June 2021

\section{Keywords}

Galgal

Rootstock identification

SCAR

Molecular characterization

\begin{abstract}
In the present investigation, seven different species specific SCAR markers were used for discriminating Galgal rootstock from other citrus species under study. The PDKV-1, PDKV-2, PDKV-3, PDKV-4, PDKV-5, PDKV-6 and PDKV-7 show the band size of 283 bp, 159 bp, 172 bp, 347 bp, 150 bp, 137 bp and 347 bp, respectively. The PIC value ranged from 0.38 to 0.88 . The highest PIC value was observed in PDKV-7 (0.88), followed by PDKV-1 (0.86) and PDKV-2 (0.83). Out of these primers, PDKV-1, PDKV-2, PDKV-6 and PDKV-7 are the potential markers for Galgal discrimination from Rangpur lime, Alemow, Jambhiri, Orange and Sweet orange. Molecular characterization of citrus species revealed highest similarity between Jambhiri and Alemow while the lowest similarity was observed between Alemow and Galgal. The cluster analysis revealed that Galgal rootstock is more diverse from other five species under study. These results will be very useful in testing the genetic purity of citrus at nursery stage.
\end{abstract}

\section{Introduction}

Citrus is one of the most economical principal fruit crops of the world. It is widely distributed throughout the tropical and subtropical regions of the world and believed to have originated in southeast Asia, particularly northeast India (Atiyah, 2016). Profitable citrus cultivation is done by grafting and budding; therefore, rootstock is a very crucial part of a citrus orchard. It has varied effects on scion vigour, fruit yield, tolerance to various biotic and abiotic stresses. It is, therefore, of requisite to select the best performing rootstock for a given variety to attain highest productivity.

Rangpur lime (C. limonia) and Rough lemon (C. jambhiri Lush.) are the broadly used rootstocks in India, due to their resistance to gummosis and root rot (Sonkar, 2001). Sometimes inadvertent seed mixtures of related species are the major obstacle for citrus grower. In India, the largest area under citrus cultivation is in the eastern region of Maharashtra where farmers totally rely on the public and private nurseries for supply of planting material. Many nurseries sadly do not maintain the mother plants of the Rangpur lime and Rough lemon rootstocks and import seeds from the Himalayan foothil states. These rootstock seeds are randomly collected from different citrus species, particularly Galgal (Citrus pseudolimon). Although, scions grafted on Galgal are vigorous and healthy. They are susceptible to Phytophthora and have a lesser life span thus, requiring replanting of the orchard after six or seven years, thus it leads to an enormous

Corresponding author: Ms. A.H. Tupke

Centre of Excellence in Plant Biotechnology, Department of Agricultural Botany, Dr. Panjabrao Deshmukh Krishi Vidhyapeeth, Akola-444104, Maharashtra, India

E-mail: anujatupke11@gmail.com

Tel.: +91-9511652112

Copyright (c) 2021 Ukaaz Publications. All rights reserved.

Email: ukaaz@yahoo.com; Website: www.ukaazpublications.com economic losses to the farmers. Therefore, a more precise system for identification of genotypes and for assessing the genetic variation in the existing germplasm is a basic requirement in citrus.

A range of methods have been used for citrus cultivar identification, like conventional method of citrus cultivar identification which relied on morphological features and isozymes. But using morphological traits, it is tough to distinguish between many citrus cultivars because some cultivars are distinguishable only by fruit traits and citrus trees usually do not bear fruits until 3-4 years after planting. Besides, isozyme markers can be mediated by secondary processes, so that the normal patterns of expression are suppressed (Atiyah, 2016). DNA fingerprinting is the innovative technique used for recognition of individual on the basis of their respective DNA profiles. It offers a rapid and more accurate way of determining relationships among closely related species than that of morphological study because morphological characteristics are subject to environmental influence (Rahman, 2007). Molecular techniques such as RAPD, RFLP, AFLP, SCAR and microsatellite markers have been used to identify citrus species with high accuracy. SCAR marker is highly reliable, co-dominant and usually single locus and species specific (Bhagyawant, 2015).

In the present study, we report the use of species specific SCAR markers to identify Galgal rootstock from five different species under study.

\section{Materials and Methods}

\subsection{Plant material}

A total six species of citrus were (Table 1) used in this study which were collected from All India Co-ordinated Research Project (AICRP) on Citrus, P.D.K.V. Akola and Central Citrus Research Institute, Nagpur. 
Table 1: List of citrus species used in the analysis

\begin{tabular}{|c|l|l|}
\hline Sr. No. & Common name & Scientific name \\
\hline 1 & Galgal & Citrus pseudolimon \\
\hline 2 & Rangpur lime & Citrus limonia \\
\hline 3 & Alemow & Citrus macrophylla \\
\hline 4 & Jambhiri & Citrus jambhiri \\
\hline 5 & Orange & Citrus reticulate \\
\hline 6 & Sweet orange & Citrus sinensis \\
\hline
\end{tabular}

2.2 Primer Designing

The species specific primers were developed from the previous sequencing results of amplified product of SCAR. Sequencing was done in bidirectional pattern from Eurofin Genomics Pvt. Ltd. Bengaluru, primer designing was carried out by using Primer Design software of NCBI BLAST.

\subsection{Primer design criteria}

The following setting was used together with the Primer design tool of NCBI BLAST.

Maximum tm difference $2^{\circ} \mathrm{C}$, minimum GC content $40 \%$ and maximum GC content $55 \%$, maximum complementarity 2 bp and maximum 32 complementarity 2 bp. Optimum Primer size 20bp and maximum primer size $23 \mathrm{bp}$. Optimum $\mathrm{Tm} 65-68^{\circ} \mathrm{C}$ in all the other entries default values were used.

\subsection{Primer production-oligo synthesis}

The designed primers were synthesized from Sigma Aldrich Bengaluru.

\subsection{DNA isolation}

Genomic DNA of six citrus species was extracted from young leaves using CTAB method as described by Amani et al. (2011). The DNA obtained by extraction was confirmed by running on $0.8 \%$ agarose gel electrophoresis system. The extracted DNA was stored at $-20^{\circ} \mathrm{C}$ until use. Concentration, quality and quantity of DNA were determined by nano drop Spectrophotometrically at $260 \mathrm{~nm}$ wavelength.

\subsection{PCR amplification}

Seven species specific SCAR primer were designed and used for identification of different citrus species. The PCR amplification was performed in a $20 \mu \mathrm{l}$ reaction volume containing $50 \mu \mathrm{g}$ of template DNA, $1 \mu 1$ of single primer, $2.5 \mu 1$ of $10 \mathrm{x}$ taq buffer $\left(\mathrm{Mgcl}_{2}\right), 1 \mu 1$ of dNTP's mixture and $0.3 \mu 1$ of taq polymerase enzyme and the remaining was filled with deionized distilled water. Amplifications was carried out as follows; initial denaturation with $94^{\circ} \mathrm{C}$ for $5 \mathrm{~min}$, followed by 35 cycles of denaturation at $94^{\circ} \mathrm{C}$ for $1 \mathrm{~min}, 65^{\circ} \mathrm{C}$ for 45 seconds for annealing and $72^{\circ} \mathrm{C}$ for $1 \mathrm{~min}$, with a final extension at $72^{\circ} \mathrm{C}$ for $10 \mathrm{~min}$. PCR products were separated by electrophoresis in $10 \%$ polyacrylamide gel with silver staining for detection. A 50-bp and 100-bp DNA ladder was used to measure the fragment size.

\subsection{Data analysis}

The amplified products were scored for the presence (1) or absence (0) of bands of various sizes across the six different citrus species to generate a binary matrix. The weak and smeared fragments were not scored. The genetic association between different species were evaluated by calculating the Jaccard's similarity coefficient based on proportion of shared bands produced by primers. The UPGMA dendrogram was constructed using Jaccard's similarity coefficient.

\section{Results}

3.1 Molecular characterization of citrus species under study, using SCAR markers

In the present investigation, seven different SCAR markers were validated for discriminating Galgal rootstock from other citrus species under study. All the seven primers are promising for discrimination of Galgal from other species under study. A total of 62 amplicons were amplified by 7 polymorphic SCAR loci and the number of amplicons ranged from 4 to 12 with an average of 8.85 amplicons per locus (Table 2). The PDKV-1, PDKV-2, PDKV-3, PDKV-4, PDKV-5, PDKV-6 and PDKV-7 show the band size of 283 bp, 159 bp, 172 bp, $347 \mathrm{bp}, 150 \mathrm{bp}, 137 \mathrm{bp}$ and $347 \mathrm{bp}$, respectively. Out of these primers, PDKV-1, PDKV-2, PDKV-6 and PDKV-7 were the best primers for discrimination of Galgal from Rangpur lime, Alemow, Jambhiri, Orange and Sweet orange. PDKV-1 was useful for discriminating Alemow species from other five species like Galgal, Rangpur lime, Jambhiri, Orange and Sweet orange at amplicon size of $510 \mathrm{bp}$. Also the band size of $90 \mathrm{bp}$ was identified as a discriminating polymorphic region for Orange species, so it discriminates orange from other five citrus species, viz., Galgal, Rangpur lime, Alemow, Jambhiri and Sweet orange (Figure 1).

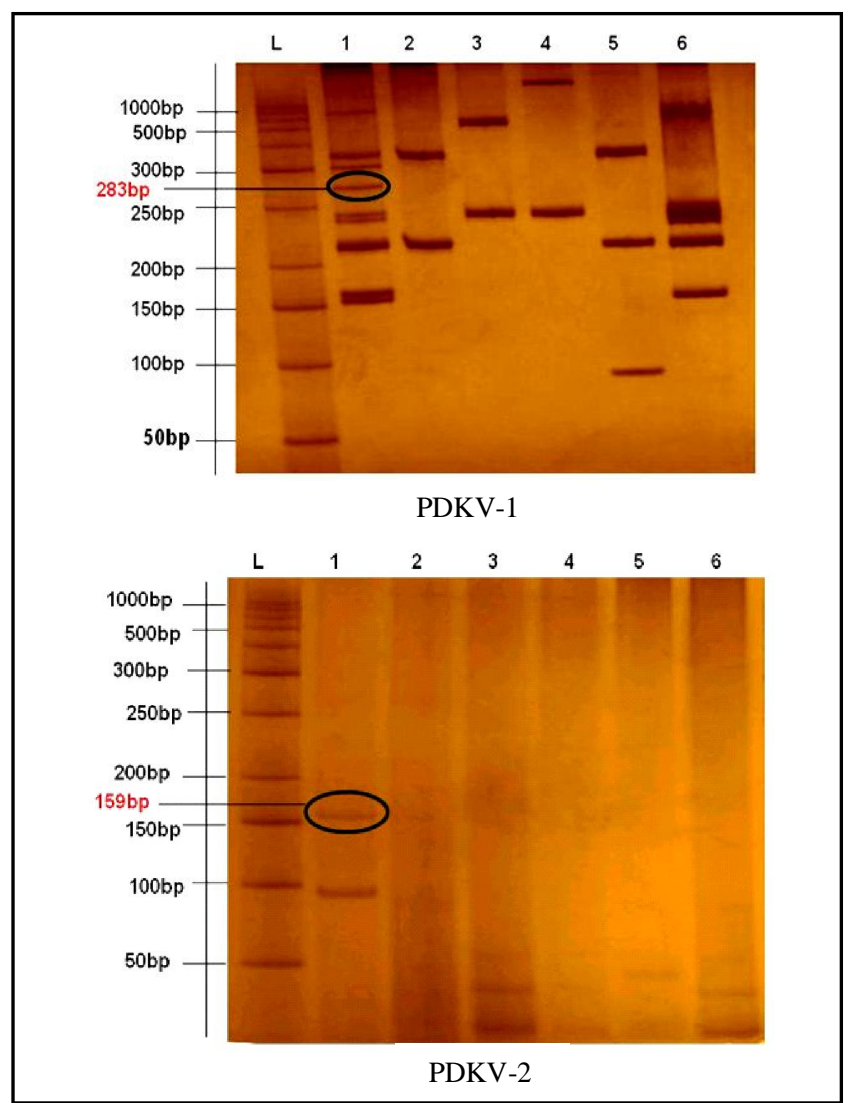

Figure 1: Species of citrus amplified with PDKV-1 and PDK V-2 Lane L-50 bp, L1-Galgal, L2-Rangpur lime, L3-Alemow, L4-Jambhiri, L5-Orange, L6-Sweet orange. 


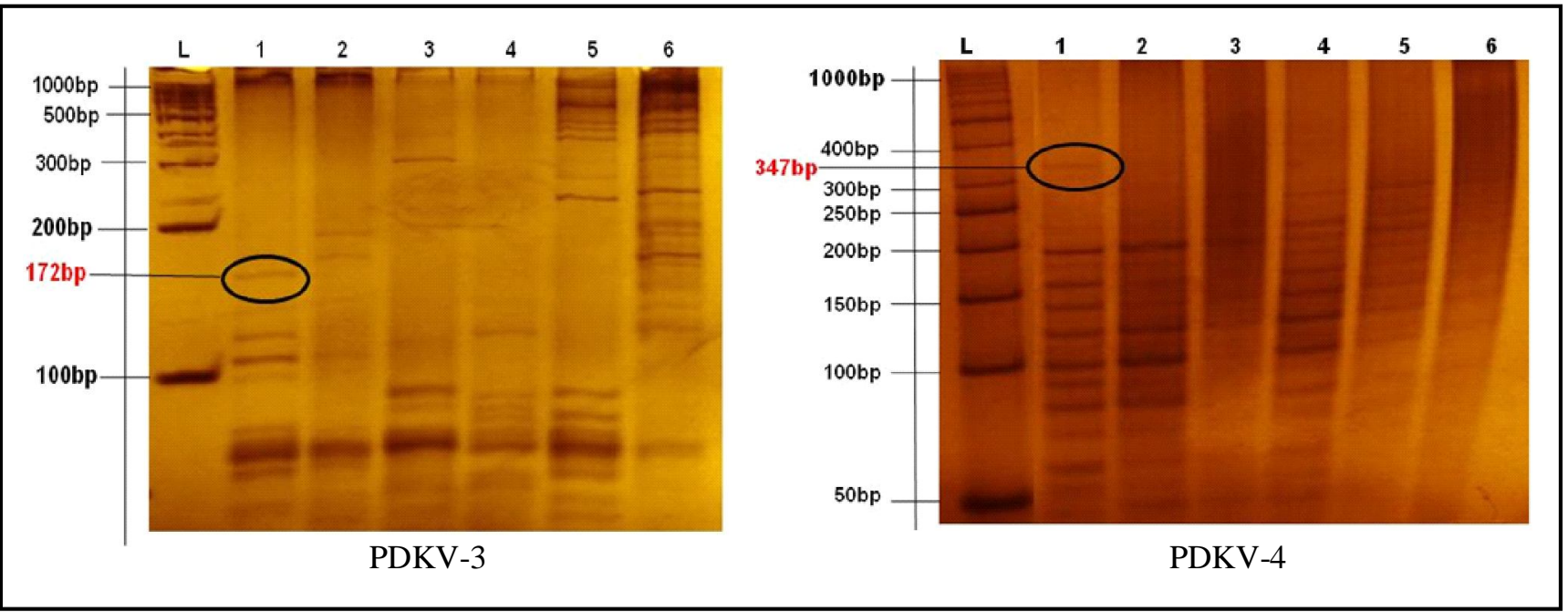

Figure 2: Species of citrus amplified with PDKV-3 and PDKV-4 Lane L-50bp, L1- Galgal, L2-Rangpur lime, L3-Alemow, L4Jambhiri, L5-Orange, L6-Sweet orange.

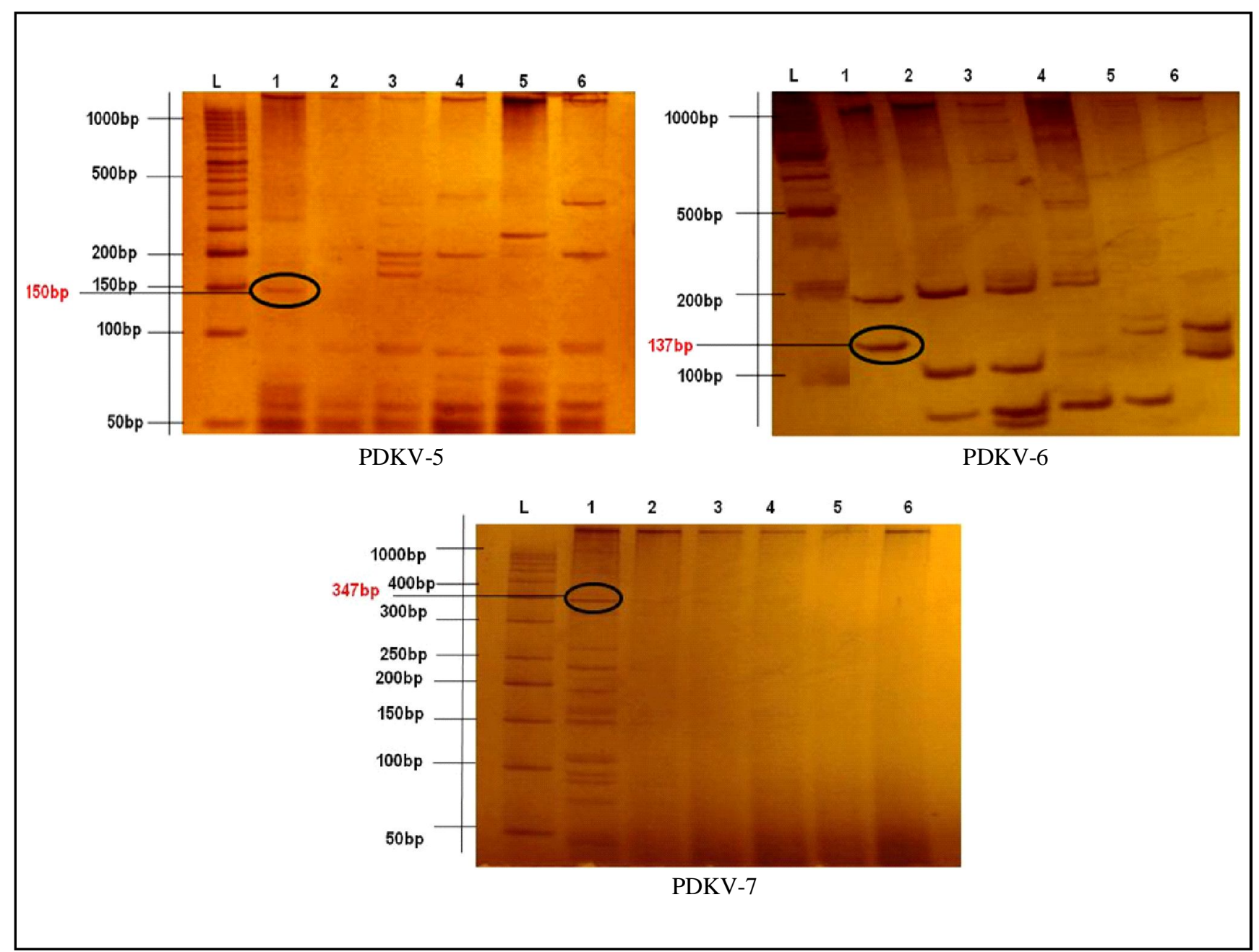

Figure 3: Species of citrus amplified with PDKV-5, PDKV-6 and PDKV-7 Lane L-50bp, L1-Galgal, L2-Rangpur lime, L3-Alemow, L4-Jambhiri, L5-Orange, L6-Sweet orange. 


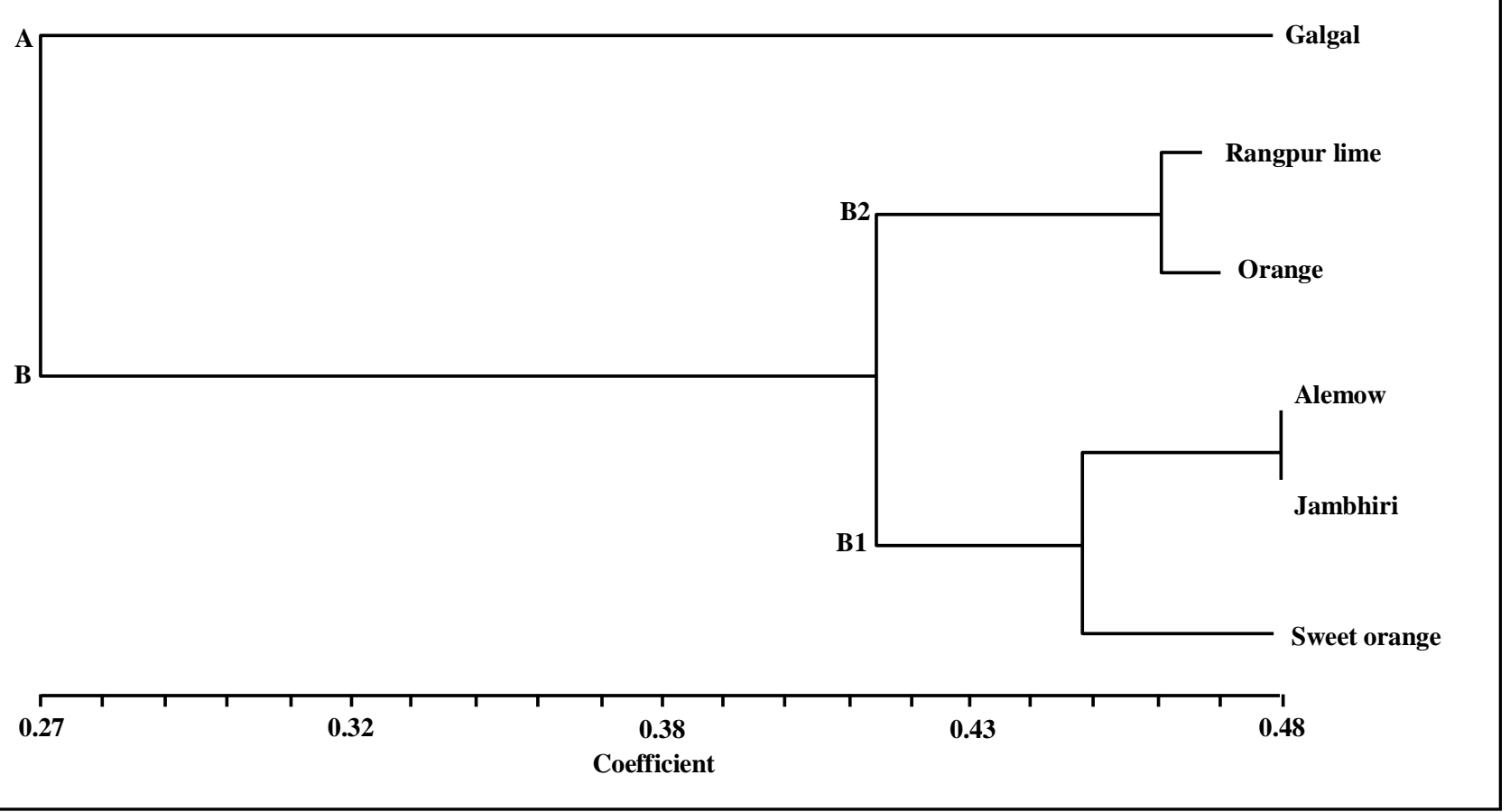

Figure 4: UPGMA dendrogram of six citrus species based on the jaccard's similarity coefficient using SCAR markers.

Table 2: SCAR primers used in the study and their PIC values

\begin{tabular}{|c|l|c|c|c|c|c|}
\hline Sr. No. & SCAR primers & No. of amplicons & Monomorphic bands & Polymorphic bands & Polymorphism (\%) & PIC value \\
\hline 1. & PDKV-1 & 12 & - & 12 & 100 & 0.86 \\
\hline 2. & PDKV-2 & 4 & - & 4 & 100 & 0.83 \\
\hline 3. & PDKV-3 & 10 & 1 & 9 & 90 & 0.73 \\
\hline 4. & PDKV-4 & 8 & 6 & 2 & 25 & 0.38 \\
\hline 5. & PDKV-5 & 10 & 2 & 6 & 75 & 0.60 \\
\hline 6. & PDKV-6 & 8 & - & 10 & 100 & 0.50 \\
\hline 7. & PDKV-7 & 10 & - & 51 & & 0.88 \\
\hline & Total & 62 & 9 & 7.28 & 84.28 & 0.68 \\
\hline
\end{tabular}

Table 3: Jaccard's similarity coefficient matrix based on SCAR markers

\begin{tabular}{|l|c|c|c|c|c|c|}
\hline Genotypes & Galgal & Rangpur lime & Alemow & Jambhiri & Orange & Sweet orange \\
\hline Galgal & 1 & & & & & \\
\hline Rangpur lime & 0.292 & 1 & & & & \\
\hline Alemow & 0.229 & 0.428 & 1 & & & \\
\hline Jambhiri & 0.272 & 0.423 & 0.482 & 1 & & \\
\hline Orange & 0.239 & 0.461 & 0.375 & 0.464 & 1 & \\
\hline Sweet orange & 0.312 & 0.375 & 0.428 & 0.468 & 0.411 & 1 \\
\hline
\end{tabular}

3.2 Polymorphic information content and per cent polymorphism

Four SCAR primers, viz, PDKV-1, PDKV-2, PDKV-6 and PDKV-7 showed $100 \%$ polymorphism followed by PDKV-3 showed $90 \%$ polymorphism with average polymorphism of all primers $84.28 \%$
(Table 2). Total alleles per locus were 8.85 , whereas average number of monomorphic and polymorphic alleles were 1.28 and 7.28, respectively. The extent of polymorphic information content (PIC) of seven primers ranged from 0.38 to 0.88 . The highest PIC value was observed in PDKV-7 (0.88), followed by PDKV-1 (0.86) and PDKV-2 (0.83). 


\subsection{Genetic diversity analysis}

SCAR markers were used to analyze the genetic diversity of citrus species. The amplicons were then scored using a 1/0 (presence/ absence) system. The Jaccard's similarity coefficient gives the extent of similarity between two genotypes. A lower similarity coefficient values indicates high diversity among genotypes. The highest similarity was found between Jambhiri and Alemow with correlation coefficient value of 0.48 . The lowest similarity coefficient was observed between Alemow and Galgal with a similarity coefficient value of 0.22 (Table 3 ).

The dendrogram constructed on the basis of molecular data. The cluster tree analysis showed that the cultivars were broadly divided into two main groups A and B with genetic similarity value reached 0.27. A group including individual one species, i.e., Galgal; B group was divided into two sub-clusters B1 and B2 with genetic similarity value reached 0.42 . The first sub-cluster (B1) was included three species; Alemow, Jambhiri and Sweet orange. A maximum similarity was observed between two species, i.e., Alemow and Jambhiri. The second sub-cluster (B2) was included two species Rangpur lime and Orange (Figure 4).

\section{Discussion}

The analysis of rootstock samples with molecular markers proved that SCAR markers were very useful and informative in the differentiation and estimation of genetic diversity within and between the different rootstocks collected from the All India Co-ordinate Research Project on Citrus, Dr. P.D.K.V. Akola and Central Citrus Research Institute, Nagpur. These results are in accordance with other studies using molecular markers to differentiate the different species of citrus (Gaikwad et al., 2013; Hvarleva et al., 2016).

PCR amplification of the genomic DNA isolated from six citrus species yielded a total of 62 amplicons were amplified by 7 polymorphic SCAR loci and the number of amplicons ranged from 4 to 12 with an average of 8.85 amplicons per locus. These results are quite similar with that of 7-15 fragments ranging per primer and with an average 10.8 fragments, reported by Hussein et al. (2003) among different citrus accessions.

The extent of polymorphic information content (PIC) of seven primers ranged from 0.38 to 0.88 . Comparable outcome was shared by Romdhane et al. (2016) and Barkley et al. (2006) where they found PIC values ranged from $0.5-0.7$ with an average value of 0.625 among different citrus accessions.

Specific alleles were identified with SCAR markers, they were able to differentiate rootstocks. The PDKV-1, PDKV-2, PDKV-3, PDKV-4, PDKV-5, PDKV-6 and PDKV-7 show the band size of 283 bp, 159 bp, 172 bp, 347 bp, 150 bp, 137 bp and 347 bp, respectively. Out of these primers, PDKV-1, PDKV-2, PDKV-6 and PDKV-7 were the best primers for discrimination of Galgal from Rangpur lime, Alemow, Jambhiri, Orange and Sweet orange. The foregoing outcome is matching with the work specified by Gaikwad et al. (2013) where they used the Sequence Tagged Microsatellite marker for identification of three citrus rootstocks namely; Galgal, Jambhiri and Rangpur lime. They found that an allele of $160 \mathrm{bp}$ was amplified in all the three genotypes. However, in Jambhiri and Rangpur lime, an additional allele of $200 \mathrm{bp}$ and $180 \mathrm{bp}$, respectively was present. So, on the basis of presence/absence of fragment, Jambhiri and Rangpur lime can be differentiated from Galgal. These primers are very useful in a breeding program since they can help to follow unique fragments in the generations and could be used as marker-assisted selection.

The clustering based on UPGMA analysis revealed the genetic variation and relationship among different species. The dendrogram showed clear cut classification of species into two different clusters. We could notice from the dendrogram, that Galgal rootstock form a separate group. The lowest similarity was observed between Alemow and Galgal with a similarity coefficient value of 0.22 . Similar results were obtained by Das et al. (2004) wherein they indicated that the 12 citrus germplasms were grouped into two major clusters likewise Uchoi (2010), Hamza (2013), Malik et al. (2013).

\section{Conclusion}

In the present study, molecular characterization of six species showed that primer-1, primer-2, primer- 6 and primer-7 are potential markers for discriminating Galgal rootstock from other species under study. Similarly, dendrogram constructed on Jaccard's similarity coefficient showed that Galgal is more diverse from other species. The genotypes categorized in different clusters can be used by breeder to develop new cultivars. Therefore, the set of SCAR markers used in present study were successful in fingerprinting and evaluating genetic diversity in the citrus species which will be of great utility for breeding of citrus germplasm.

These results will be very useful in testing the genetic purity of citrus seedling at nursery stage.

\section{Conflict of interest}

The authors declare that there are no conflicts of interest relevant to this article.

\section{References}

Amani, J.; Roohallah, K.; Ali, R.A. and Ali, H.S. (2011). A simple and rapid leaf genomic DNA extraction method for polymerase chain reaction analysis. Iranian J. of Biotechnology, 9(1):69-71.

Atiyah, A.S. (2016). Molecular characterization and genetic diversity analysis of sweet Orange (Citrus sinensis L. Osbeck) cultivars in Iraq using RAPD markers. European Journal of Molecular Biotechnology, (11), Is. 1 .

Gaikwad, A.; Archak, S. and Saxena, S. (2013). Technology development for identification of Citrus (Citrus spp.) rootstocks based on Sequence Tagged Microsatellite marker. Indian Journal of Agricultural Sciences, 83(10):1110-1112.

Badiyala, S.D. and Sharma, S.K. (2004). Variability studies in Galgal (Citrus pseudolimon Tanaka) in upper shivaliks of Himachal Pradesh. Indian Journal of Horticulture, 61(2):105-108.

Bhagyawant, S.S. (2015). RAPD- SCAR markers: An interface tool for authentication of traits. Journal of Biosciences and Medicines, 4:1-9.

Barkley, N.A.; Roose, M.L.; Krueger R.R. and Federici, C.T. (2006). Assessing genetic diversity and population structure in a Citrus germplasm collection utilizing simple sequence repeat markers (SSRs). Theor. Appl. Genet., 112:1519-1531.

Gaikwad, K.A.; Patil, S.R.; Nagre, P.K. and Potdukhe, N.R. (2018). Morphological characterization of Citrus rootstock genotypes. International Journal of Chemical Studies, 6(2):516-529.

Hamza, E. M. (2013). Genetic diversity of some citrus varieties based on microsatellite and RAPD molecular markers in Egypt. World Journal of Agricultural Sciences, 9(4):316-324. 
Hussein, H.A.; Samir, M.M.; Nahala, A.A. and Hussein, M.S. (2003). Genetic analysis in some citrus accessions using microsatellites and AFLP based markers. Arab. J. Biotech., 6(2):203-222

Hvarleva, T.; Kapari-Isaia, T.; Papayiannis, L.; Atanassov, A.; Hadjinicoli A. and Kyriakou, A. (2016). Characterization of Citrus cultivars and clones in Cyprus through microsatellite and RAPD analysis. Biotechnology and Biotechnological Equipment, ISSN: pp:1310-2818.

Malik, S.K., Chaudhury, R.; Dhariwal, O.P. and Kalia, R.K. (2006). Collection and characterization of Citrus indica Tanaka and C. macroptera Montr.: Wild endangered species of northeastern India. Genetic Resources and Crop Evolution, 53(7):1485-1493.

Malik, S.K.; Uchoi, A.; Kumar, S.; Choudhary, R.; Pal, D.; Kole, P.R.; Chaudhury, R. and Bhat, K.V. (2013). Molecular characterization of Citrus macroptera Mont. (Satkara): An endangered wild species from northeast India. Plant Biosystems, 147(4):857-863
Rahman, O.R. (2007). DNA fingerprinting in Utricularia L. section Utricularia as revealed by PCR based assay. International Journal of Botany, 3(1):56-63.

Rattanpal, H.S.; Singh H. and Uppal, G.S. (2018). Genetic divergence in trifoliate Citrus rootstocks under sub-tropical conditions of Punjab. Journal of Pharmacognosy and Phytochemistry, 7(1):953-957.

Romdhane, M.B.; Riahi, L.; Selmi, A. and Zoghlami, A. (2016). Molecular characterization and genetic relationships among Tunisian Citrus rootstocks. Journal of New Sciences, 32(2):139-145.

Sonkar, R.K.; Singh S. and Naqvi, S.A.M.H. (2001). Citrus rootstocks. In: Citrus, (Eds.) International Book Distribution Co. Lucknow, India, pp. 119-132.

Uchoi, A.; Malik, S.K.; Choudhary, R.; Kumar, S.; pal, D.; Rohini, M.R. and Chaudhury, R. (2017). Molecular markers in assessing genetic variation of Indian citron (Citrus medica L.) cultivars collected from different parts of India. Indian Journal of Biotechnology, 2(16):346-356. 